Journal of Materials and Environmental Sciences ISSN : 2028-2508 CODEN : JMESCN University of Mohammed Premier

OujdaMorocco

\title{
Preparation and study of properties of polyvinyl chloride-scrap tire composite for civil applications
}

\author{
Mahmood M. Barbooti ${ }^{1^{*}}$, Bushra H. Musa ${ }^{1}$ Q.A. Salloom ${ }^{2}$, D. Abdul-Aziz ${ }^{2}$ \\ ${ }^{1}$ Department of Applied Science, University of Technology, Sinaa Street, P.O. Box 35045, Baghdad, Iraq. \\ ${ }^{2}$ Directorate of Materials Research, Ministry of Science and Technology, Jadiriyah, Baghdad, Iraq
}

Received 29May 2017,

Revised O1Sep 2017,

Accepted 10Sep 2017

Keywords

$\checkmark$ scrap tires,

PVC,

$\checkmark$ Thermal insulator,

$\checkmark$ composites,

$\checkmark$ Mechanical

properties

M.M. Barbooti

brbt2m@gmail.com

$+9647502415259$

\begin{abstract}
Scrap tires, SCT, represent an environmental problem due to their durability and rigidity as they are designed to suit heavy duty road applications. Many attempts were employed on semi-industrial and pilot scales to find possible uses for the scrap tires as supply of energy and materials via thermochemical conversion and inclusion is some composite materials to suit practical applications. In this work, thermoplastic polymer matrix (PVC), with the incorporation of SCT particles, will be used to prepare composite material with reliable thermal insulation. The composites were processed using a twin-screw extruder. The thermal and mechanical properties of the prepared composites with SCT contents of $10-40 \%$ were evaluated. Young's modulus decreases with the increase in the SCT content and a linear relation with $\mathrm{R}^{2}$ of 0.936 describes the effect. Both the Shore $\mathrm{D}$ hardness and tensile strength linearly decreased with the increase in SCT powder content. The impact strength increased linearly with the increase of SCT particles content of the composite. The increase of the SCT content in the composites resulted in a decrease of the. The study indicated the possibility of using scrap tires at as much as $30 \%$ in the production of thermal insulation sheets with acceptable mechanical properties and reasonable thermal insulation characteristics. The thermal conductivity values of the prepared composites ranged from $0.24-0.29 \mathrm{~W} / \mathrm{m}{ }^{\circ} \mathrm{C}$ which is common for thermal insulators.
\end{abstract}

\section{Introduction}

The massive disposal of SCT and the huge expansion of their stockpiles in dumping sites created an ideal environment for the growth of rats and mosquitoes with their bad consequences on life [1]. Fire hazard is another potential source of scrap tire disposal problems [2]. The main task in SCT utilization is the reduction of the huge quantities of such a highly engineered material that suits the tough application being running on roads. The production of tires involves the incorporation of many ingredients to end up with a strong modified performance material. Such perfection in their production makes the natural degradation more difficult and hence efficient methodologies have to be introduced to solve the problem. An estimate of the annual accumulation of scrap tires approached 1.5 billion tires [3]. The SCT have been used in a variety of rubber and plastic products, burning for production of electricity, or as fuel for cement kilns, and as modifiers in asphalt concrete $[4,7]$. The use of SCT in building components, particularly in Portland cement concrete, has become popular application in the last few decades. Great effort is directed for the utilization of SCT as a source of liquid fuels and chemicals [8-11]. Due to crosslinked structure of rubber vulcanizates, pyrolysis seems to be the most effective way for tire recycling. In a pyrolysis reactor the shredded tires are decomposed into pyrolysis products: solid char (30-40 wt\%), liquid residue (50-60 wt\%), and gases (3-20 wt \%) [12].

Many pyrolysis systems were introduced on the bench and pilot scales to decompose the shredded materials at various temperatures employing a variety of geometries for the feeding of the material and the collection of the products [10]. In addition to the usual hydrocarbons, the degradation of the backbone rubber material may result in the formation of some valuable products like lanoline as a result of the dimerization of the produced monomers [13]. 
Aside from such techniques, material scientists and engineers started to include the SCT in some useful products to be used in various applications. The use of such products in building industry appears to be a viable route for contributing to both preventing environmental pollution and designing economical buildings. The increase in the popularity of using environmentally friendly, low cost and light weight construction materials in building industry brings the need for searching more innovative, flexible and versatile composites. The various products can be utilized in other applications like highway guard rail extensions, railroad tie pads, paving stones, and floor coverings of at least about one inch in thickness [14], for making roofing products such as rolled roofing, tiles, shakes, slates, and walkway pads [15].

Yesilata, et al., [16] studied and characterized concrete samples loaded with the SCT and other polymeric wastes utilizing the adiabatic hot-box technique for comparing effective thermal transmittances of the samples. The addition of such materials into concrete significantly reduced the heat loss and, thus, improved the thermal insulation performance. The degree of improvement in thermal insulation is found to vary with the added waste material and geometry of shredded-pieces.

A method for forming foamed thermal insulating material from scrap rubber was disclosed by Disanto [17] by mixing Scrap buffed rubber with a flour, water and yeast mixture, a deodorant, an antivermin agent and a flame retardant, and baked to form a cellular material suitable for thermal insulation. Waste rubber powder was incorporated into thermoplastic polymers with the objective to improve their properties. For instance, waste rubber powder has been incorporated with linear low density polyethylene (LLDPE) to obtain impact-resistant thermoplastic elastomer, TPE [18].Plesuma and Malers [19] prepare a binder from recycled tires and polyurethane to work in water medium

PVC is the most used polymer for variety of general purposes domestic, industrial applications and for wiring and cable insulation and sheathing. Colom et al., 2007, [20] studied the compatibility between PVC and other recycled polymers including SCT to be used as reinforced composite materials. Nikpour [21] utilized SCT powder as an impact modifier for natural fiber-polymer composites.

SCT Rubber is a thermoset material which makes the recycling a difficult practice and a trend to use it in mixtures with thermoplastic matrixes to suit industrial and household applications was used. The lack of adhesion of SCT rubber with the rest of polymers makes this practice not possible [22].

The blends of thermoplastic materials with SCT powders exhibit properties typical of rubbery materials but can be processed like thermoplastics. They can be easily processed by internal mixing or extrusion, and their productivity is high, because no vulcanization is required. Besides, these types of blends provide better material utilization as scrap and rejects can be recycled [23].

The present work aims at the preparation of PVC composites with various contents of SCT to suit the thermal insulation applications and reliable mechanical properties.

\section{Experimental details}

\subsection{Materials}

Poly vinyl Chloride (PVC) powder was purchased from Sabic, Saudi Arabia. Lead stearate, Zinc stearate, and Dioctyl phthalate (DOP) were supplied from Guangdong Winner Co. The SCT powder was supplied by the State Company for Tire Industries, Najaf, Iraq. The powdered material was sieved and the fraction of (0.6-1.18 $\mathrm{mm}$ ) particle size was used in this study.

Lead stearate was used as a stabilizer in PVC and also acts as a high temperature lubricant. Zinc stearate (white powder), with a density of $1.095 \mathrm{~g} / \mathrm{cm}^{3}$ and melting point $(120-130)^{\circ} \mathrm{C}$, was employed as a releasing agent and external lubricant which can be easily incorporated. Dioctyl phthalate (DOP) acts as a plasticizer and added in a ratio of $10 \%$ for all preparations.

\subsection{Procedures:}

\subsubsection{Preparation of PVC Matrix}

The ingredients were added in the following order: PVC powder, stabilizer, zinc stearate, and then the lead stearate. Dimethyl dichlorosilane was used as a coupling agent and was added as ethanol solution to SCT rubber and left for $24 \mathrm{hrs}$ for drying and then heated at $200^{\circ} \mathrm{C}$ and left for 2 days for complete drying. The SCT rubber was ready for the next steps of composite preparation.

\subsubsection{Preparation of polymer composite}

The SCT rubber powder was added to the prepared PVC and during mixing, the DOP was added gradually to the mixture. The Composites were prepared with $10,20,30$, and $40 \%$ by weight of SCT mixed with PVC. The mixing was carried out in a two-roll mill machine (made in China for Areejul-Furat Company - Iraq) at (80$110^{\circ} \mathrm{Cfor} 10 \mathrm{~min}$. The samples were then extruded by a locally produced twin screw extruder at a screw speed of 
$50 \mathrm{rpm}$.To produce polymer composite with $4-\mathrm{mm}$ thickness, stainless steel mold of $(10 \mathrm{~cm} \times 10 \mathrm{~cm} \times 0.4 \mathrm{~cm})$ in dimensions was used. The extrudate was compressed with hydraulic press at $\left(150^{\circ} \mathrm{C}\right)$ and a pressure of 7 ton for $10 \mathrm{~min}$. The product was cooled in a water bath and removed from the mold. Finally, the samples were properly shaped by mechanical aids in accordance with the standard specifications to fulfill the mechanical tests in the work.

\subsection{Physical and Mechanical Tests}

\subsubsection{Tensile tests}

The tensile test samples were dumbbell-shaped and were cut according to the standard ASTM-D638 dimensions of $100 \times 20 \times 4 \mathrm{~mm}$ at room temperature. The measurements were carried out on a Tinus Olsen tensile strength system, model H50KT. During the measurements the course of the load and elongation were timely illustrated on the screen. Tensile strength was calculated by the relation [24].

$$
\text { Tensiles strength }=\frac{\text { Load at break }}{\text { (original width )(original thickness })}
$$

Stress at break, ultra tensile strength, UTS, was evaluated from the following equation:

$$
\tau_{\mathrm{b}}=\frac{F}{A}
$$

Where, $A$ is the cross - sectional area of the sample and $F$ is the applied force. The elongation at break was calculated using the following equation:

$$
\Delta L_{\mathrm{b}}=\frac{\left(\mathrm{L}^{\prime}-\mathrm{L}_{\mathrm{o}}\right)}{\mathrm{L}_{\mathrm{o}}} \times 100
$$

Where, $\dot{L}$ is the length of sample at break and $L_{0}$ is the initial length. The modulus of elasticity was evaluated using the following equation:

$$
\varepsilon=\frac{\tau_{\mathrm{b}}}{\left(\mathrm{L}^{\prime}-\mathrm{L}_{\mathrm{o}}\right) / \mathrm{L}_{\mathrm{o}}}
$$

where, $L$ is the length of sample at yield, and $\tau y$ is the stress at yield.

\subsubsection{Charpy impact tests [25]}

The Charpy impact test was carried on sample of $55 * 10 * 4 \mathrm{~mm}$ in accordance with the ISO 79 standard. Some of this kinetic energy is utilized to cause the fracture the sample. The impact strength (I.S) was calculated by applying the relationship:

$$
\mathrm{I} . \mathrm{S}=\mathrm{U}_{\mathrm{c}} / \mathrm{A}
$$

where $\mathrm{U}_{\mathrm{c}}$ is the fracture energy (Joule) which is determined from Charpy impact test instrument, A: is the crosssectional area of the specimen.

\subsubsection{Hardness test}

The hardness is measured by Shore (D) Durometer device model TH210, Italy, in accordance to (ISO 9001) standard. The hardness value is determined by the penetration of the Durometer indenter foot into the sample by applying a sufficient pressure for 5 seconds.

\subsubsection{Bending test [26]:}

A Three-points tester purchased from Phywe, Germany was used to measure the Young's modulus of samples. The bending test was performed according to ASTM-D790 standard on sample $100 \mathrm{~mm}$ in length, $10 \mathrm{~mm}$ in width and $5 \mathrm{~mm}$ height. The Young's modulus $\left(\mathrm{E}_{\mathrm{b}}\right)$ is calculated from the Eq. (5):

$$
E_{b}=\frac{M g L^{3}}{48 I S}
$$

$(\mathrm{M} / \mathrm{S})$ is the slope calculated from mass-deflection curve; $\mathrm{g}$ is the acceleration due to gravity $\left(9.81 \mathrm{~m} / \mathrm{s}^{2}\right)$; and I is the momentum of geometrical bending. The latter is calculated from the equation:

$$
I=\frac{b d^{3}}{12}
$$


where $b$ is the width $d$ is the thickness and $L$ is the distance between supports, all been measured in meters.

\subsubsection{Thermal conductivity}

Lee's disc instrument was used to determine the thermal conductivity of the samples under test. It consists of three discs of brass and a heater. The heat transfers from the heater to the next two discs then to the third disc across the sample. A voltage, $\mathrm{V}$, of $6 \mathrm{~V}$ was applied to the heater at a current, I, of $0.25 \mathrm{~A}$. The temperatures of the discs $\left(T_{A}, T_{B}\right.$, and $\left.T_{C}\right)$ were recorded with the thermometers located in them at equilibrium. The values of the thermal conductivity were calculated using the equation:

$$
I . V=\pi r^{2} e\left(T_{A}+T_{B}\right)+2 \pi r e\left[d_{A} T_{A}+d_{S} \frac{1}{2}\left(T_{A}+T_{B}\right)+d_{B} T_{B}+d_{C} T_{C}\right]
$$

where, $r$ is the radius of the sample, $d_{A}, d_{B}$ and $d_{C}$ are the thickness of discs $A, B$ and $C$, respectively; $d_{S}$ is the thickness of the sample and $e$ is the heat flowing through the cross sectional area of the sample per unit time $\left(\mathrm{W} / \mathrm{m}^{2} .{ }^{\circ} \mathrm{C}\right)[24]$. The thermal conductivity, $K\left(\mathrm{~W} / \mathrm{m} .{ }^{\circ} \mathrm{C}\right)$, can then be calculated $[28,29]$ from equation $(8)$ :

$$
K\left(\frac{T_{B}-T_{A}}{d_{S}}\right)=e\left[T_{A}+\frac{2}{r}\left(d_{A}+\frac{1}{4} d_{S}\right) T_{A}+\frac{1}{2 r} d_{S} T_{B}\right]
$$

\subsubsection{Flexural Strength}

Properties of materials, such as fracture resistance and elasticity, under stress are evaluated by the determination of properties of flexural strength, flexural modulus, and fracture toughness [30]. While the failure stress of a material is called flexural strength, the stiffness of a material is called flexural modulus as both measured in bending [31]. The flexural strength of the composite is determined from 3-point bend test.

Fresh fruits of Malusdomesticawere bought from King Fahd University of Petroleum and Minerals (KFUPM) commercial shopping centre. They were thoroughly washed thrice with double distilled water, sliced into pieces, dried in a hot air oven at $70{ }^{\circ} \mathrm{C}$ for $24 \mathrm{~h}$ and then crushed into powder using a grinder (Joya, 16-002). An intense brown colourMalusdomestica extract was obtained after mixing $10 \mathrm{~g}$ of the fruit powder in $200 \mathrm{~mL}$ double distilled water and then boiling it for $10 \mathrm{~min}$ in a hot plate. The residue was separated by filtration through Whatman No. 1 filter paper. The filtrate obtained was stored in a refrigerator for further use.

\section{Results and Discussion}

\subsection{Mechanical properties}

The mechanical properties of the prepared composites were evaluated by carrying out a number of tests like: young's modulus, shore D hardness, tensile strength, impact strength, flexural strength, and thermal conductivity.

\section{Bending test}

Fig. 1 illustrates the effect of SCT content of the composite on the Young's modulus, $\mathrm{E}_{\mathrm{b}}$, of the composite. It is clear that Young's modulus decreases with the increase in the SCT content. Almost linear correlation could be obtained with $\mathrm{R}^{2}$ of 0.936 . Consequently, the values of $\mathrm{E}_{\mathrm{b}}$ of composites decreased. The presence of SCT improved the rigidity, although the compatibility is not sufficient. The elastomer phase is uniformly dispersed particles in the PVC matrix which was bearing the tensile force applied on the blends. These findings are in agreement with previous work [32]. Agglomeration and particle-particle interaction of the rubber powder were observed decrease in tensile strength and Young's modulus of blends. The molecular entanglements in the rubber chains alone are insufficient to prevent rapid flow and fracture in response to the applied stress. This results in the lower tensile strength and Young's modulus of the PVC composite. For HDPE/GTR blends, the presence of the crosslinking rubber powder and others curatives in GTR has allowed the rubber particles to reach higher strains and at the same time confers mechanical strength to the particles [32].

\subsubsection{Hardness test}

Fig. 2 shows the change of the Shore D hardness of the composites with SCT content. A linear decrease of the Shore D hardness was obtained with the increase in SCT powder content of the composite with a reasonable correlation coefficient, $\mathrm{R}^{2}$, of 0.9582 . The hardness decrease indicated a decrease in the crosslinking density of the blends. The incorporated SCT introduced flexibility and elasticity of the rubber chains into PVC matrix. It also resulted in less rigid rubber vulcanizates with a decrease in hardness [33]. This observation is in perfect agreement with the results on Young's modulus. 


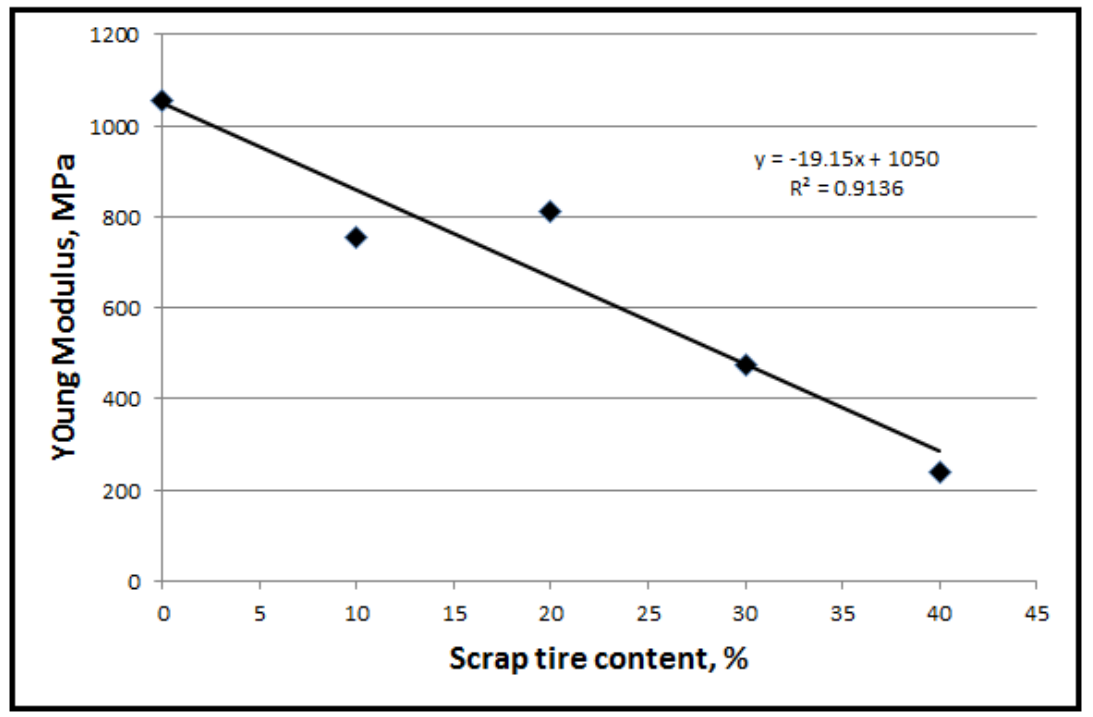

Figure 1: The effect of waste tire on the young modulus of PVC composite.

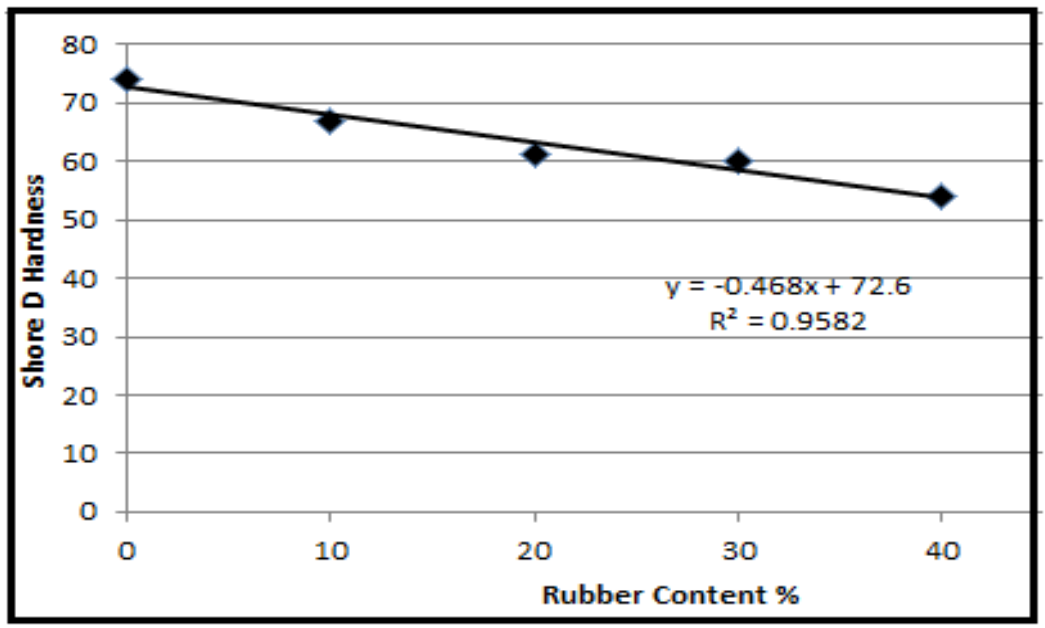

Figure 2:The change of Shore D hardness with SCT content of the composites.

\subsubsection{Impact strength}

Fig. 3 shows the impact strength values of the composite as a function of the SCT content. The impact strength increases linearly with the increase of SCT particles content of the composite. The PVC matrix plasticity provides ductility to the composite, whereas scrap tire will cause more brittle behavior with a subsequent loss of toughness in the composite material [34]. An increase in the filler SCT content in matrix resulted in an increase in the impact strength of the specimens. For scrap tire contents beyond $30 \%$ the increase of the impact strength is only slightly increased.

\subsubsection{Tensile test}

Fig. 4 shows the plot of tensile strength versus the waste rubber addition. The increase of the SCT content in the composites resulted in a decrease of the tensile strength. The rate of decrease was almost linear with a correlation coefficient of 0.9303 . The relation of the SCT content with the tensile strength can be represented with the equation (9):

Tensile Strength $=13.86-0.2351($ SCT $\%)$

Such a decrease of tensile modulus may be associated with the aggregation of filler particles at high concentration. The tensile modulus has the same trend as crosslink density which is measured from the swelling measurement. However, strong matrix-filler interaction due to the increase of the number of crosslinks between polymer chains will cause a loss of flexibility of the polymer composite. Thus, value of elongation at break shows a reduction with increasing waste rubber loading and gives the opposite trend of tensile modulus resulted in composites becoming stiffer and harder. As a result, the thermoplastic polymer composites can be processed 
by extrusion, injection, and compression molding. The results were in agreement with those reported by Montagna and Santana [34].

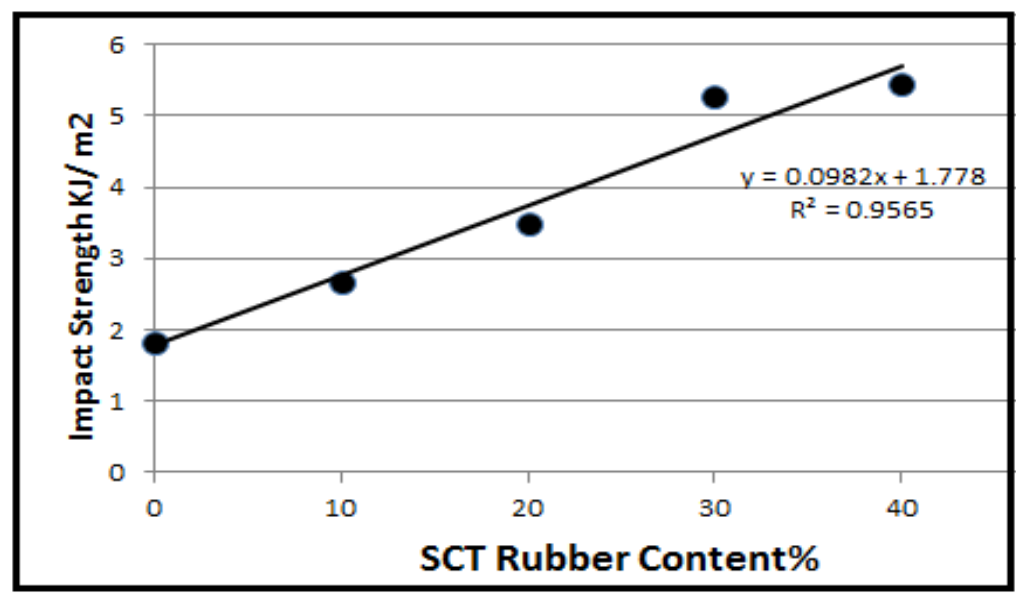

Figure 3: The impact strength values of the composite as a function of the scrap tire content.

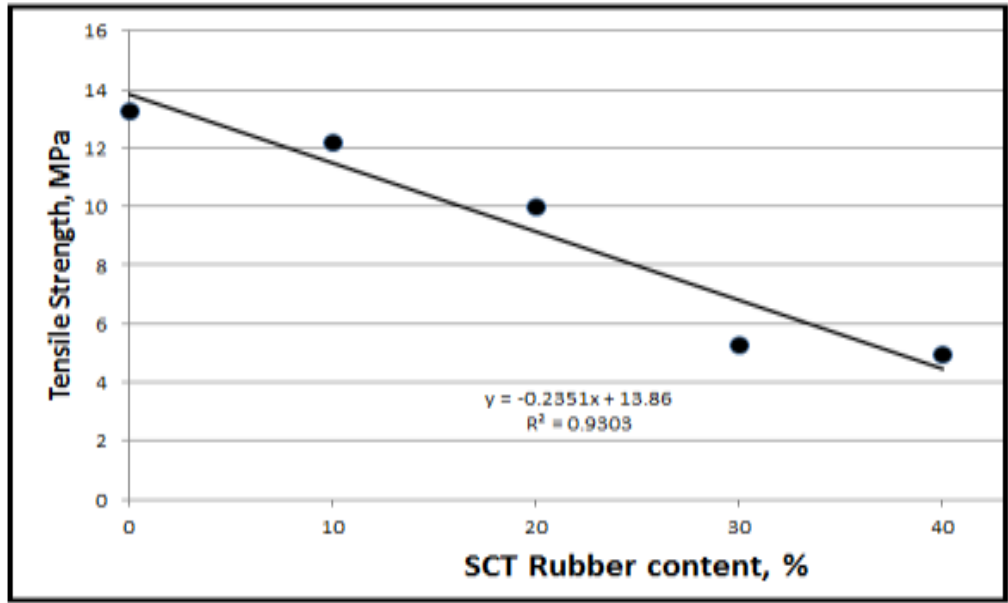

Figure 4: The variation of tensile strength versus the waste rubber addition.

Thus, the more ground tire rubber incorporated in the PVC matrix the lower the mechanical properties will be. This is mainly due to the lack of adhesion with thermoplastic matrixes, the particle size of the ground tire rubber particles and its cross-linked nature [33].

The reduction in strength may be due to agglomeration of the SCT particles to form a domain that acts like a foreign body as well as weak interfacial region. Similar trend of tensile strength was observed by Ismail et al. [29] in their work on white rice husk ash filled natural rubber composites.

\subsubsection{Flexural Strength}

Composite designers highly depend on flexural strength because composite resins, especially cavities under stress, are exposed to tension and compression forces [30, 35]. Fig. 5 shows the dependence of the flexural strength values on the SCT content of the PVC - SCT composite. It is clear that the composite showed an increase in the flexural strength as the SCT content increases and attains a maximum value of about $35 \mathrm{MPa}$ at about 15\% SCT content. Further addition of SCT resulted in more flexible material and this is expected because of the flexibility of the SCT particles. Similar trend could be obtained by Reddy et al., [36] in their study if enforcement of epoxy resin with glass fiber.

\subsubsection{Thermal conductivity measurements:}

The effects of SCT content of the composite on the thermal conductivity of PVC are shown in Fig. 6. The thermal conductivity decreased from 0.35 for the pure PVC down to a minimum value of about 0.24 for the composite with $10 \%$ wt ratio of SCT powder. This best thermal insulation could be obtained at the $10 \%$ wt ratio of the SCT powder. There appears an increase in the thermal conductivity of composite with the increase of scrap tire content. This may be a result of the presence of some thermally conductive components within the 
scrap tire composition like carbon black and zinc oxide and silica. The rate of increase was lower with scrap tire content above $20 \%$ and values of 0.2905 and $0.2933 \mathrm{~W} / \mathrm{m} .{ }^{\circ} \mathrm{C}$ for the 30 and $40 \%$ scrap rubber contents, respectively. The conductivity may result from the expected touching of many filler particles with each other to begin to form filler conductive chains, which greatly contribute to the thermal conductivities of composites.

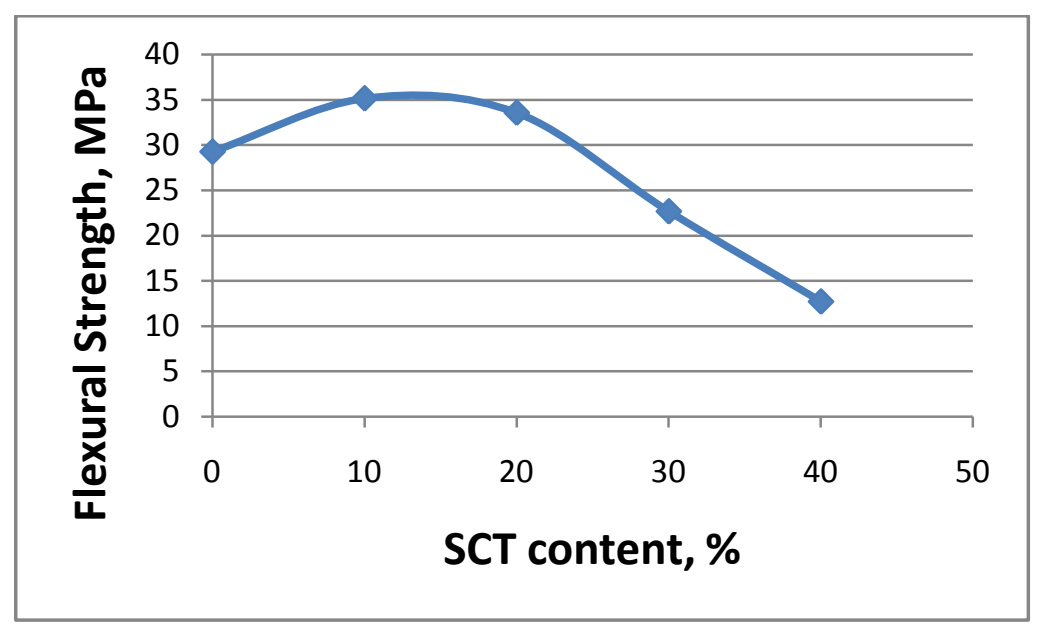

Figure 5:Flexural strength values of the PVC - SCT composite.

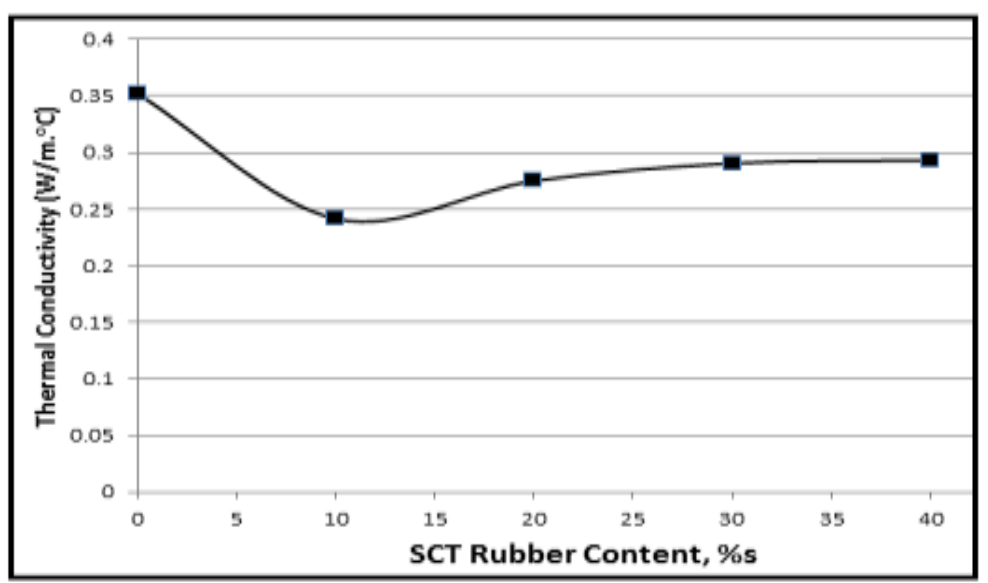

Figure 6: The dependence of thermal conductivity on the scrap tire content of the composite.

When the thermal conductivity of the $20 \%$ of the scrap tire content, $0.25 \mathrm{~W} / \mathrm{m} \mathrm{K}$, is compared with those of some common construction materials, it was less than the thermal conductivity of clay bricks [13], the wood concrete [18] and concrete containing waste rubber [19]. Thus, conductivity values of our samples fall within the range of insulating materials.

\section{Conclusion}

Thermally insulating composites with reliable mechanical properties can be prepared by incorporating scrap tire powder into PVC matrix at ratios of 20-30\%. The achieved thermal conductivity of the prepared composites compares well with clay bricks, wood concrete. The preparation is performed by normal machinery and no specific conditions are required.

Acknowledgement-The authors are grateful to Moayyadfaik, and SuadQasim and ZAID MAJID for carrying out the mechanical tests.

\section{References}

1. K.D. Sadhan, I.I. Avraam, K. Klementina, Rubber Recycling, New York: CRC press, (2005) 300-301.

2. N.M. Nikpour, M. Sc. Thesis, Laval Univ., Québec, Canada, 2016.

3. M. Nehdi and A. Khan, Cem. Concr. Aggr; 23 (2001) 3-10.

4. R. Siddiqu and T.R. Naik, Waste Manage; 24 (2004) 563-9.

5. S. Raniand R. Agnihotri, Recycling of scrap tires, Internat. J. Mater Sci. Appl. 3(5) (2014) 164-167. 
6. D. Shao, M. Xu, L.Cai, and S.Q.Shi, Fabrication of Wood-Rubber Composites Using Rubber Compound as a Bonding Agent Instead of Adhesives, Materials, 9, (2016) 469

7. C. Karakurt, Microstructure properties of waste tire rubber composites: an overview, J Mater Cycles Waste Manag (2015) 17:422-433 DOI 10.1007/s10163-014-0263-9

8. M.M. Barbooti, T.J.Mohammed, A.A.Hussain,and F.O.Abas, J. Anal. Appl. Pyrolysis, 72 (2004) 165- 170.

9. C.F.S. Rombaldo,A.C.L. Lisbôa; M.O.A. Méndez,A.R.Coutinho, ,Mat. Res. 11(3) (2008) 359-363

10. M.M. Barbooti, Iraqi Patent, No. 4542, on Apr. 224, 2016. Int. Classif. B01D3/00; B09B3; C08J11/12; C10G1/10

11. M.M. Barbooti, J. Anal. Appl. Pyrolysis, 110 (2014) 419-423.

12. M. Juma, Z. Koreňová, J.Markoš,J.Annus, and L. Jelemenský,Petroleum and Coal 48 (1) (2006) 15-26.

13. B. Danon, P.van der Gryp, C.E.Schwarz, and J.F.Gorgens, J. Anal. Appl. Pyrolysis, 112 (2015) 1-13.

14. W. H. Frankland, U.S. Pat. No. 4,244,841, March 24, 1980.

15. S.S. Lalwani, W. G.Pursell, Sr., ,C. J.Horner, Jr., , US. Pat. No. 4,851,500.

16. B. Yesilata, Y.Isıker,andP.Turgut, Construction and Building Materials 23 (2009) 1878-1882.

17. R.J. di Santo, US Pat. 4174308 A, 1979.

18. P.K. Pramanik, and Baker, W.E., Plast. Rubber Comp. Process. Appl., 24 (1995) 229.

19. X. Colom, X. Cañavate, F. Carrillo, M. Mañosa, M.J. Lis, Annual Internat. Conf. Composites /Nano Enginnering, Presentation's date 2010-07-04.

http://wjoe.hebeu.edu.cn/sup.2.2010/BC/Colom,\%20X.\%20_Barcelona,\%20Spain_\%20\%20129.pdf.

20. R. Plesuma, L. Malers, Functional properties of composite material from recycled tires and polyurethane binder in water medium, Baltic Polymer Symposium 2015 IOP Publishing IOP Conf. Series: Materials Science and Engineering 111 (2016) 012004 doi:10.1088/1757-899X/111/1/012004.

21. L.Scheirs, Polymer Recycling. Chichester : Willey \& Sons , 1998, 412-413.

22. P.C.Carné, Study of compatibilization methods for High Density Polyethylene and Ground Tire Rubber: Exploring new routes to recycle scrap tyres, Ph. D. Thesis, Univ. Catalonia Barcelona, 2009.

23. M.A.Lopez-Manchadoand M.Arroyo, Polymer, 4(1) (2000) 776.

24. J.K.Oleiwi, E.S.Al- Hassani, andA.A.Mohammed, ,Eng. Tech. J., Part (A), 32(3) (2014) 653-666.

25. R.J.Crawford, Engineering, Plastics, $2^{\text {nd }}$ Edn.,Pergamon Press, New York, (1987).

26. W.D.Callister, Material Science and Engineering, New York, 1999.

27. D.M.Price and M.Jarratt, , Proc. 28th Conf. North Amer. Thermal Anal. Soc., Orlando, 2000.

28. A.M.Collieu and D.J.Powney, The Mechanical and Thermal Properties Butter and Tanner, London (1973).

29. G.Tolf and P.Clarin,Fiber Composite Hybrid Materials Applied Science Publisher LTD, London, (1981).

30. J. Manhart, K.H. Kunzelmann, Chen HY, Hickel R, Dent Mater 16 (2000) 33-40.

31. A.U. Yap and S.H.Teoh,J Oral Rehabil 30(2003) 171-177.

32. H. Ismail, and H.Suryadiansya, Polymer Testing, 21(4) (2002) and 389-395. http://dx.doi.org/10.1016/S0142-9418(01)00101-5.

33. A.Puşcă, Ş.Bobancu and A.Duţă,Bull. Transilvania Univ. Braşov, 3 (2010) 52.

34. L.S.Montagna, and R. M. C.Santana,Plastics, Rubber and Composites, 41(6) (2012 ) 256- 262.

35. N. Eronat, U. Candan, M.Türkün, J EsthetRestor Dent 21 (2009) 171-178.

36. S.P. Reddy, P.V.Chandra, S.Rao, A. C.Reddy, and G. Parmeswar, Internat. Conf. Adv. Mater. Manufact. Technol. (AMMT) Dec. 2014, Hyderabad, PP 98-102.

(2018) ; http://www.jmaterenvironsci.com 